\title{
Feasibility of Self-administered Neuromodulation for Neurogenic Bladder in Spinal Cord Injury
}

\author{
Argyrios Stampas ${ }^{1,2}$, Rose Khavari ${ }^{3}$, Joel E. Frontera ${ }^{1,2}$, Suzanne L. Groah ${ }^{4}$ \\ ${ }^{1}$ Department of Physical Medicine and Rehabilitation, McGovern Medical School, UTHealth at Houston, Houston, TX, USA \\ ${ }^{2}$ TIRR Memorial Hermann, Houston, TX, USA \\ ${ }^{3}$ Department of Urology, Houston Methodist Hospital, Houston, TX, USA \\ ${ }^{4}$ Department of Rehabilitation Medicine, MedStar Georgetown University Hospital, Washington, DC, USA
}

\begin{abstract}
Purpose: To determine if self-administered transcutaneous tibial nerve stimulation (TTNS) is a feasible treatment option for neurogenic bladder among people with spinal cord injury (SCI) who utilize intermittent catheterization for bladder management.

Methods: Four-week observational trial in chronic SCI subjects performing intermittent catheterization with incontinence episodes using TTNS at home daily for 30 minutes. Those using anticholinergic bladder medications were given a weaning schedule to begin at week 2 . Primary outcomes were compliance and satisfaction. Secondary outcomes included change in bladder medications, efficacy based on bladder diary, adverse events, and incontinence quality of life (I-QoL) survey.

Results: All 16 subjects who started the study completed the 4-week trial rating TTNS with high satisfaction and easy to use, without discomfort. Twelve of 14 patients (86\%) using anticholinergic bladder medications reduced their dosage and maintained similar frequency and volumes of bladder catheterization and incontinence episodes. Bladder medication reduced by approximately $3.2 \mathrm{mg}$ weekly ( $95 \%$ confidence interval, -5.9 to -0.4 ) and anticholinergic side effects of dry mouth and drowsiness decreased more than 1 level of severity from baseline ( $\mathrm{P}=0.027, \mathrm{P}=0.015$, respectively). At 4 weeks, total I-QoL score improved by an average of 3.2 points compared to baseline in all domains.

Conclusions: This pilot trial suggests TTNS is feasible to be performed at home in people with chronic SCI. Participants were able to reduce anticholinergic medication dosage and anticholinergic side effects while maintaining continence, subsequently improving QoL scores. These results advocate for further randomized, controlled trials with longer duration and urodynamic evaluation to assess long-term efficacy.
\end{abstract}

Keywords: Spinal cord injuries; Neuromodulation; Neurogenic bladder; Transcutaneous electric stimulation; Anticholinergic

- Grant/Fund Support: This research was funded by TIRR Memorial Hermann Rehabilitation Innovation Grant Award 2017. Research effort is supported by Mission Connect, a project of the TIRR Foundation.

- Research Ethics: The study was performed according to the Helsinki Declaration (http://www.wma.net/en/30publications/10policies/b3/) and approved by the Institutional Review Board (IRB) of UTHealth (HSC-MS-17-0423) where the experiment was performed. Written informed consent was obtained from all subjects.

- Conflict of Interest: No potential conflict of interest relevant to this article was reported.

Corresponding author: Argyrios Stampas (iD https://orcid.org/0000-0003-4600-6208 TIRR Memorial Hermann, 1333 Moursund St, Houston, TX 77030, USA E-mail: argyrios.stampas@uth.tmc.edu / Tel: +17137975938 / Fax: +17137997052 Submitted: July 4, 2019 / Accepted after revision: August 5, 2019
This is an Open Access article distributed under the terms of the Creative Commons Attribution Non-Commercial License (http://creativecommons.org/licenses/by-nc/4.0/) which permits unrestricted non-commercial use, distribution, and reproduction in any medium, provided the original work is properly cited. 


\section{INTRODUCTION}

There are over 1 million people living with spinal cord injury (SCI) in the world resulting in neurogenic bladder (NGB) in nearly all who have suprasacral injuries [1,2]. NGB in SCI has long been recognized as a major contributor to morbidity and reduced quality of life (QoL), identified as the number one research priority in those living with SCI in 2004 [3]. Revisiting the research priorities 15 years later, NGB remains the top priority in those living with SCI [4]. Indeed, the current management options are suboptimal, with up to $50 \%$ of people with SCI NGB developing serious urologic complications [5].

The goal for bladder management in SCI is often intermittent catheterization [6]. After conservative approaches have failed, the current treatment approach to reduce incontinence between catheterization is to initiate overactive bladder $(\mathrm{OAB})$ medications, which can inhibit detrusor overactivity (DO), increase bladder capacity, and decrease detrusor pressures [2]. This medication management suffers from a high rate of noncompliance among people with SCI due to the anticholinergic side effects (i.e., dry mouth, sedation, constipation, etc.) where larger doses are often required to prevent urinary incontinence $[7,8]$. Furthermore, anticholinergic medications are limited as a long-term treatment as newer evidence suggests an inherent risk of dementia with higher cumulative anticholinergic medication doses $[9,10]$. Alternative treatment options without increasing anticholinergic burden are necessary.

One such alternative is chemodenervation of the bladder using onabotulinum toxin $\mathrm{A}$, which has shown efficacy in treating NGB in SCI [11]. However, there are several limitations to consider, including the expensive and invasive cystoscopy required, the need for annual repeated treatments, and the increased risk of urinary tract infections (UTIs), the most frequently reported adverse event occurring in $24 \%-56 \%$ of cases $[12,13]$. Neuromodulation is another US Food and Drug Administration (FDA) approved treatment option for OAB which does not have anticholinergic side effects, although considered experimental in the NGB population.

Neuromodulation of the bladder evolved from the work of Tanagho and Schmidt in the 1980s, in which they were able elicit voiding with electric stimulation of the ventral sacral nerve roots $[14,15]$. This research led to the development of a surgically implanted electronic device for sacral neuromodulation (SNM), FDA-approved for symptoms of OAB in 1997 and more recently, received FDA approval for the treatment of fecal incontinence in 2010. SNM has shown efficacy in the SCI population, but had considerable surgical complications [16]. This surgical procedure is expensive and largely unavailable to the SCI population. With far less risks and adverse events, percutaneous tibial nerve stimulation (PTNS) is another FDA-approved neuromodulation treatment for OAB.

Tibial nerve stimulation (TNS) was first described by McGuire et al. [17] in 1983, using electric stimulation of the posterior tibial nerve to inhibit detrusor activity, as seen in hind limb stimulation in animal models. This was then translated to human clinical trials and since the 1990s, PTNS has received FDA approval for the treatment of OAB. PTNS requires needles to be placed by trained healthcare professionals through the skin of the inner ankle to stimulate the tibial nerve [18]. Benefits of PTNS include increased bladder capacity, reduced detrusor pressure, and improved QoL [19,20]. Delivered weekly in clinic, PTNS has low compliance and is not a feasible treatment option in a population known to have difficulty accessing health care $[18,21]$. However, neuromodulation has the potential to hurdle the barriers in NGB management in patients with mobility impairments by developing a noninvasive, readily-available, inexpensive treatment that can be used at home.

Consider transcutaneous TNS (TTNS), the transcutaneous adaptation of PTNS. PTNS has demonstrated equal efficacy to newer anticholinergic bladders medications in a randomized control trial of 100 SCI subjects, while boasting fewer adverse events [22]. A large study of this nature has not been performed with TTNS in SCI. However, in a multicenter study of NGB due to multiple sclerosis, TTNS improved urinary urgency in more than $80 \%$ of the subjects, reduced frequency, and had a positive impact on QoL measures [23]. To investigate whether TTNS could be used from acute injury to home, we performed TTNS in acute SCI and found it safe and feasible, with evidence suggesting efficacy [24]. The observed significant decline in bladder capacity and increased detrusor-sphincter dyssynergia (DSD) in the sham-control group was mitigated in the TTNS group [24]. We next sought to test whether TTNS could be feasibly self-administered in the community setting in people with paraplegia.

\section{MATERIALS AND METHODS}

This clinical trial was approved by the Institutional Review Board and is registered with clinicicaltrials.gov: NCT03458871. From March 2018 to January 2019, subjects who presented for 
routine follow-up to our tertiary care SCI clinic were screened and recruited for our study. Inclusion criteria for eligible subjects were individuals older than 18 years of age with a traumatic, chronic SCI ( $>1$ year), and using intermittent catheterization to empty their bladders with complaints of incontinence between catheterizations. Excluded were individuals with active neurologic and medical issues, other genitourinary diagnoses (i.e., benign prostate hypertrophy, bladder cancer), symptomatic UTI, neurologic levels of injury caudal to thoracic level 9 (T9), more than 2 medications for hyperreflexive bladder, changes in these medications in the past month, and onabotulinum toxin A treatment for NGB in the prior 6 months.

After meeting initial screening criteria, subjects interested in participating in the study had TTNS performed in clinic to demonstrate proper placement of electrodes. TTNS was applied to the right leg with the negative electrode behind the internal malleolus and the positive electrode $10 \mathrm{~cm}$ superior to the negative electrode, verified by great toe flexion or toe spreading with rising current intensity (Fig. 1). Subjects were excluded if we could not verify that the tibial nerve was being electrically stimulated.

Once enrolled, baseline incontinence quality of life (I-QoL) survey and an anticholinergic side effect questionnaire were administered. Known anticholinergic side effects (dry mouth, constipation, hard stool, sleepy/tired, decreased thought process, and decreased sexual drive) were scored as none, mild, moderate, or severe. These surveys were repeated after the 4-week trial, with an additional TTNS satisfaction survey consisting of a continuous scale of $0=$ strongly disagree, $5=$ neutral, and $10=$ strongly agree.

Subjects were instructed to perform TTNS with frequency of $10 \mathrm{~Hz}$ and 200- $\mu \mathrm{sec}$ duration with current intensity lowered until absence of toe movement for 30 minutes at constant stimulation daily for 4 weeks [24-26]. For those that were using bladder medications, they were individually provided with a weaning schedule. They were to use TTNS concurrently with their medications for week 1 . At week 2 , they were to reduce one of their bladder medications in half, and if tolerating this reduction, continue reducing medications weekly. At any point, if patients perceived increased urinary frequency or increased incontinence episodes, they were to contact the study team to increase their dosage and attempt weaning the following week.

Subjects were sent home with the protocol instructions, a commonly prescribed NMES (neuromuscular electric stimulator) device (Stamobil+, Innovative R P Inc., Houston, TX, USA) with 5 pairs of electrodes (1 pair per week), and a bladder diary for the 4-week trial. In the bladder diary, subjects were instructed to record daily catheterizations and volumes, bladder incontinence (accidents/leaks), and milligrams of daily bladder medication. They also recorded the use of TTNS with current intensity settings and whether or not they achieved toe flexion. All UTIs, occurrence of skin irritation, or any other change in medical statues required phone contact with the study team. The research assistant contacted subjects weekly to collect bladder diary data and monitor participants' progress and compliance.

\section{Statistical Methodology}

For this pilot trial, a convenience sample of 16 subjects was used. For compliance and satisfaction with TTNS, descriptive statistics were calculated to determine the dropout rate and percent of adherence to the prescribed regimen as well as program satisfaction, respectively. For evaluation of QoL changes during the study period, change in mean I-QoL scores were calculated from posttrial and baseline survey. Changes in catheterization measures and anticholinergic bladder medications were compared with average weekly measures using Wilcoxon matched pair signed rank or paired t-test. Mixed-effects linear regression modeling was also used with the bladder diary variables to eval-
Procedure:

- Toe flexion to verify proper position

-30 minutes of daily use

- Weekly electrode replacement

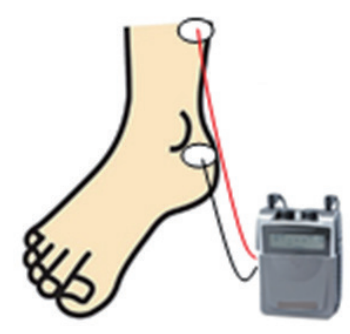

Protocol:

- Baseline and 4-week surveys

- Daily voiding diary for 4 weeks

- Week 1: TTNS+no change in OAB meds

- Week 2-4: TTNS+medication weaning weekly as tolerated

Fig. 1. Brief description of TTNS procedure and protocol with an illustration of the TTNS surface electrode placements near the ankle and at the distal leg. TTNS, transcutaneous tibial nerve stimulation; OAB meds, overactive bladder medications. 
Table 1. Baseline characteristics

\begin{tabular}{|c|c|}
\hline Characteristic & Value \\
\hline Age $(y r)$, mean \pm SD & $38.9 \pm 11.1$ \\
\hline Years of injury, median (IQR) & $8.75(3.1-19.4)$ \\
\hline Male sex & $13(82)$ \\
\hline $\begin{array}{l}\text { Bladder medication } \\
2 \text { Medications } \\
1 \text { Medication } \\
0 \text { Medication }\end{array}$ & $\begin{array}{r}3(19) \\
11(69) \\
2(13)\end{array}$ \\
\hline $\begin{array}{l}\text { Race } \\
\text { White } \\
\text { Hispanic } \\
\text { Black } \\
\text { Asian }\end{array}$ & $\begin{array}{l}6(38) \\
6(38) \\
2(12) \\
2(12)\end{array}$ \\
\hline $\begin{array}{l}\mathrm{AIS}^{\mathrm{a})} \\
\mathrm{A} \\
\mathrm{B} \\
\mathrm{C} \\
\mathrm{D}\end{array}$ & $\begin{array}{l}11(69) \\
2(13) \\
2(13) \\
1(6)\end{array}$ \\
\hline $\begin{array}{l}\text { Neurologic level }^{\mathrm{b})} \\
\text { C1-4 } \\
\text { C5-8 } \\
\text { T1-4 } \\
\text { T5-9 }\end{array}$ & $\begin{array}{l}0(0) \\
3(19) \\
6(37) \\
7(44)\end{array}$ \\
\hline $\begin{array}{l}\text { Etiology } \\
\text { MVA } \\
\text { GSW } \\
\text { Other }\end{array}$ & $\begin{array}{r}10(63) \\
4(25) \\
2(12)\end{array}$ \\
\hline
\end{tabular}

Values are presented as number (\%) unless otherwise indicated. SD, standard deviation; IQR, interquartile range; AIS, American Spinal Injury Association Impairment Scale; MVA, motor vehicle accident; GSW, gunshot wound.

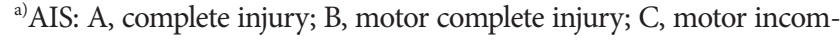
plete injury with less than half of muscles below the motor level with antigravity strength; $D$, motor incomplete injury with at least half of the muscles below the motor level with antigravity strength. ${ }^{\text {b) Neurologic }}$ level: C, cervical; T, thoracic. uate the changes over time adjusting for subject variability.

\section{RESULTS}

\section{Compliance and Satisfaction (Section I)}

Of the 21 subjects screened in clinic, all 16 subjects that consented to participate completed the trial. Baseline characteristics are presented in Table 1. Mean age was 38.9 years (standard deviation [SD], 11.1) and median duration of injury was 8.8 years (interquartile range, $3.1-11.1$ years). About $80 \%$ of the subjects were male, consistent with the increased prevalence of men with SCI. There were 3 subjects with tetraplegia (19\%), 11 with complete injury (69\%), and 14 (88\%) were using anticholinergic bladder medications. Of these, 3 (21\%) used 2 bladder medications. All 16 subjects completed the 4-week trial. Aside from lower genitourinary tract adverse events (some worsening incontinence described in section III), no other adverse events were reported, including skin problems, burns, autonomic dysreflexia, changes in bowel function, and changes in sexual function. Fourteen of the 16 subjects recorded data in the bladder diary which showed $98 \%$ compliance for daily use in the 4 -week period. The remaining 2 subjects verbally attested to daily use but were not used for this analysis. All 16 subjects completed the satisfaction survey at the end of the 4-week trial. All subjects reported no pain from the use of TTNS (Fig. 2). Fourteen of the 16 subjects strongly agreed (10 of 10) that TTNS was easy to use and 15 of 16 would strongly agree (10 of 10) to recommend it to others.

\section{Quality of Life (Section II)}

Baseline and posttrial I-QoL and anticholinergic side effect scores are presented in Fig. 3. Mean changes in I-QoL total and

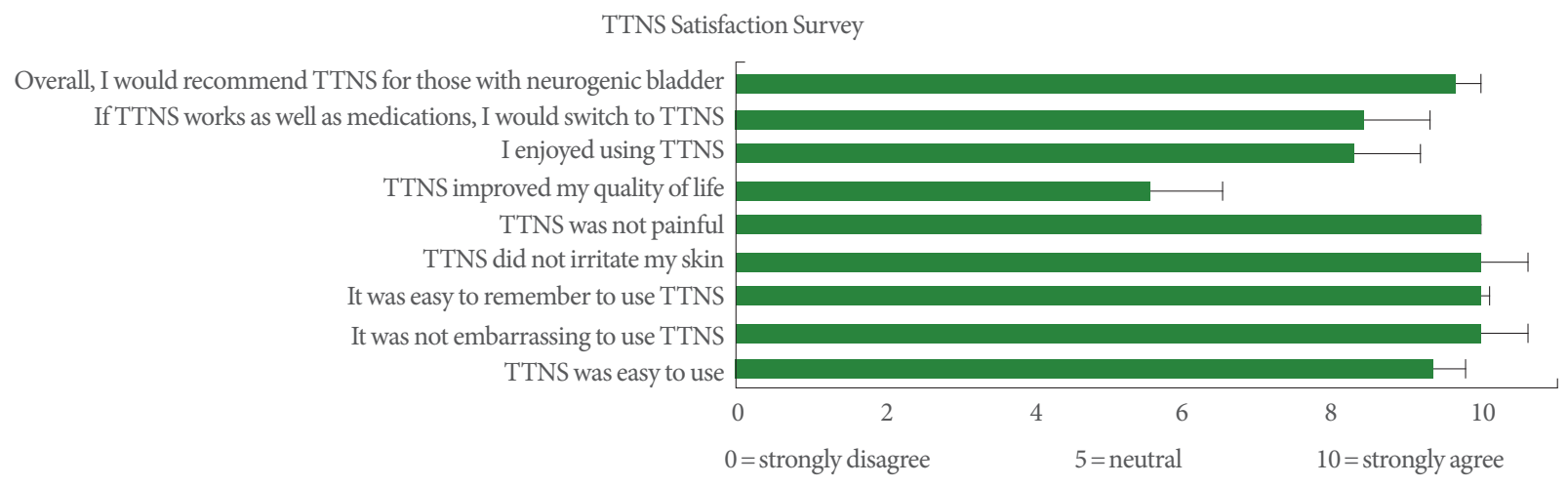

Fig. 2. Mean responses with standard error bars to questions on transcutaneous tibial nerve stimulation (TTNS) Satisfactions Survey. 

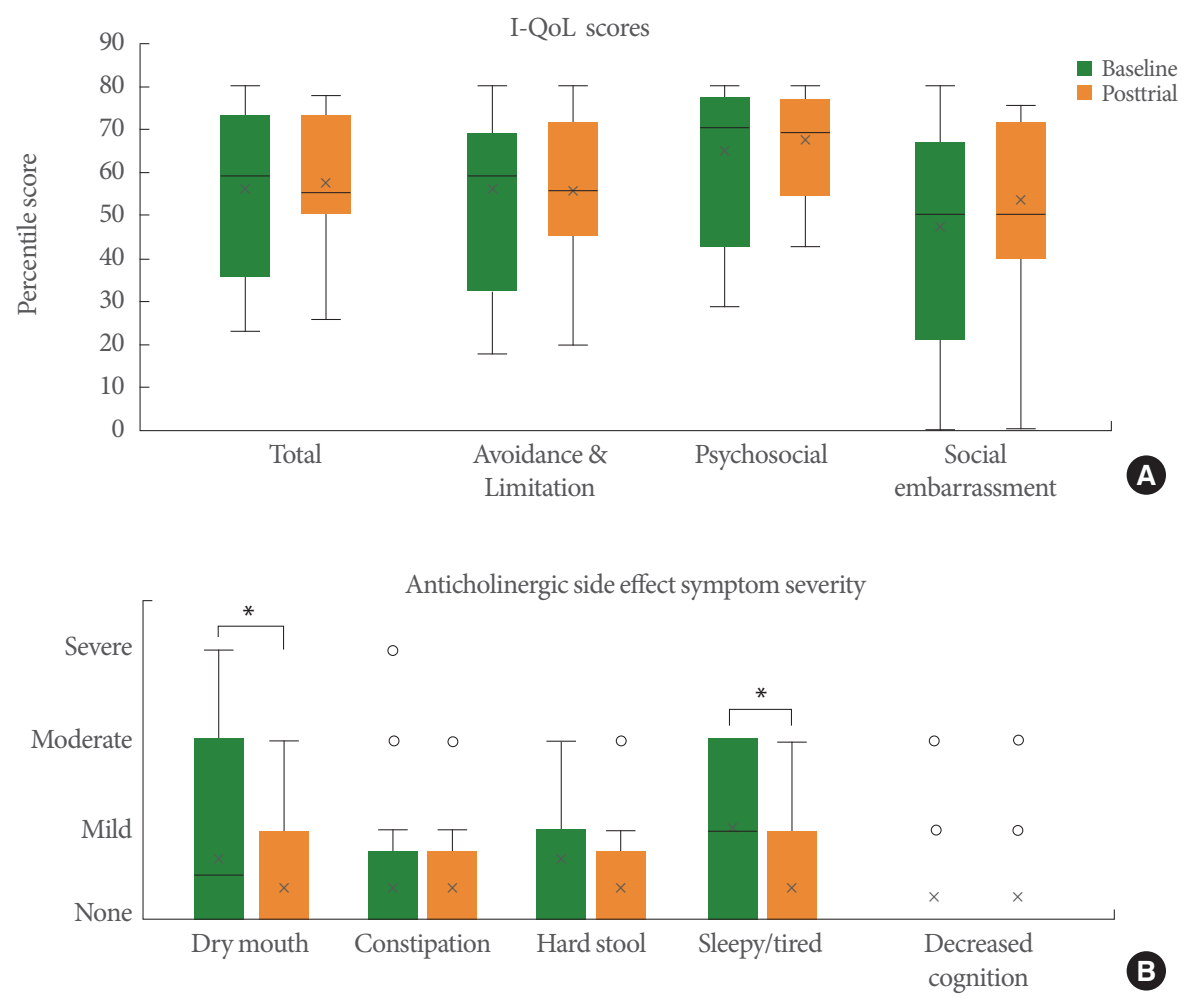

Fig. 3. (A) Baseline and posttrial results of incontinence quality of life (I-QoL) survey total and subscores. (B) Baseline and posttrial responses to anticholinergic side effect severity of symptom questionnaire. ${ }^{*} \mathrm{P}<0.03$.

subscores were greater than 2 points, defined as "a little better" by the I-QoL scoring instructions. The mean change in the severity of anticholinergic side effect symptoms based on the questionnaire decreased significantly by 1.5 categories $(-1.5$, $\mathrm{P}=0.01$ ), indicating less anticholinergic side effects. Specifically, dry mouth severity reduced from severe to moderate (6 [38\%] to $1[6 \%], P=0.027)$ and sedation severity reduced from severe to mild (10 [63\%] to 6 [38\%], P=0.015) after the 4-week TTNS trial. Demographics and SCI phenotypes were not significantly associated to I-QoL and anticholinergic side effect scores.

\section{Catheterization and Anticholinergic Bladder Medication Measures (Section III)}

Weekly average catheterization frequency and volumes, as well as occurrence of bladder incontinence episodes (accidents/ leaks), were stable over the 4 weeks, despite significant reductions in milligrams of bladder medications (Fig. 4). Most subject $(8,57 \%)$ reported stable or decreased episodes of average weekly incontinence episodes. Those with more average weekly incontinence episodes from baseline $(>1)$ had: discontinued their bladder medications entirely ( 1 of $6,17 \%)$; were taking 2 bladder medications ( 3 of $6,50 \%$ ); and had symptomatic UTIs (2 of $6,33 \%)$.

Of the 14 subjects using bladder medications at the beginning of the trial, $12(86 \%)$ were able to reduce their bladder medications, 4 (29\%) were able to completely discontinue one bladder medication including one subject using 2 medications. Mean reduction of bladder medication dosage was $8.6 \mathrm{mg}$ from baseline (mean \pm SD, 26.6 $\pm 25.0 \mathrm{mg}$ ) to the end of the 4-week trial (18.0 \pm $23.4 \mathrm{mg}$ ). Based on mixed-effects linear regression modeling adjusting for subject variability, bladder medication dosage decreased by $3.2 \mathrm{mg}$ per week of TTNS use (95\% confidence interval, -5.9 to -0.4$)$. The 2 participants that were not able to reduce their bladder medications were using 2 bladder medications and experienced increasing incontinence episodes with dose reductions. Intensity of stimulation and occurrence of toe flexion did not change significantly over the 4-week study period.

\section{DISCUSSION}

Supported by strong prospective observational and brain imaging studies regarding the association between anticholinergic 


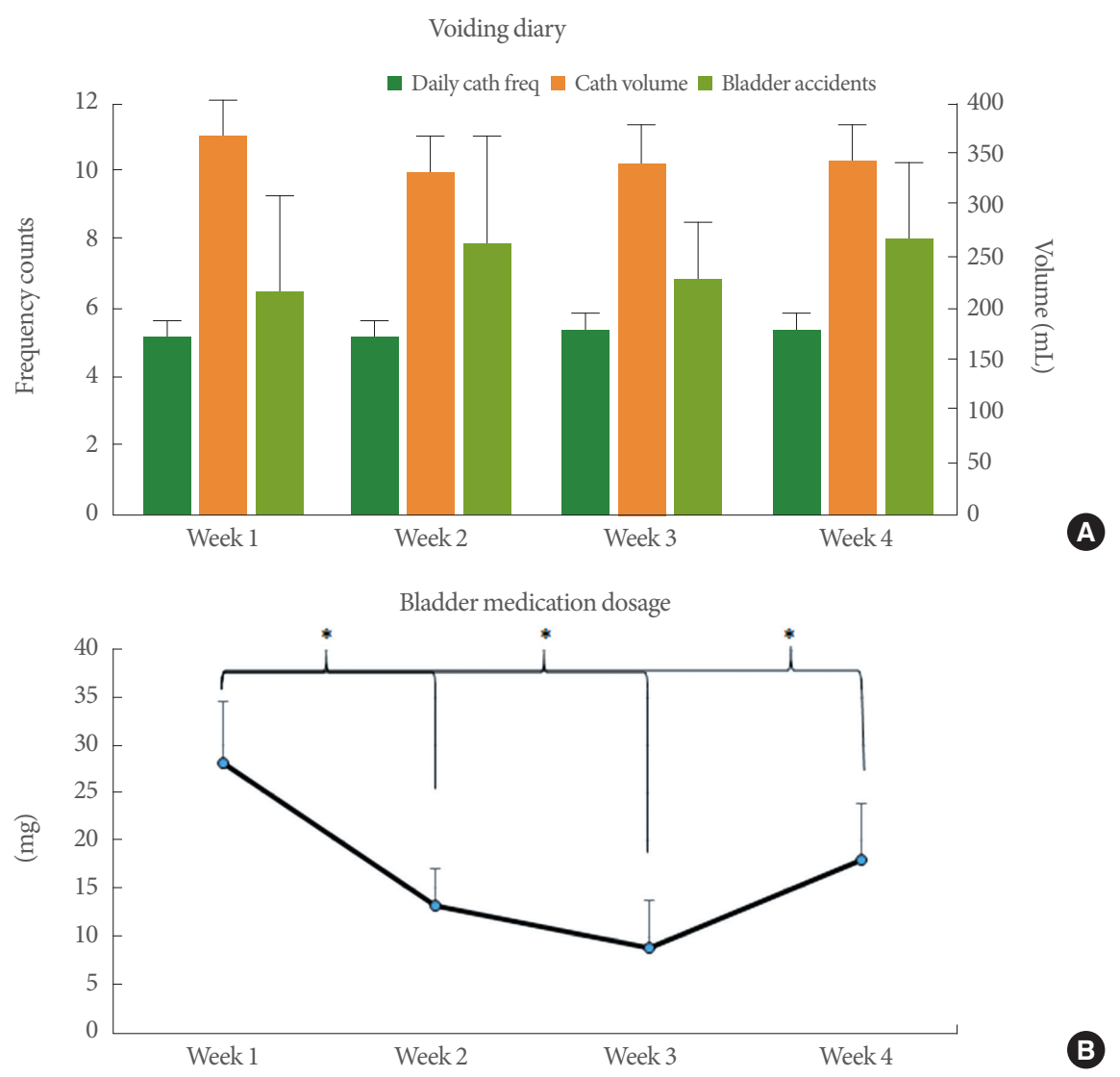

Fig. 4. (A) Weekly voiding diary demonstrate stable volumes of catheterization (cath), frequency (freq) of daily catheterizations, and bladder incontinence (accidents). (B) Significant reductions in mean weekly milligrams of anticholinergic bladder medications. ${ }^{*} \mathrm{P} \leq 0.003$.

medication use and cognitive impairment, Alzheimer disease, and dementia, the American Urogynecologic Society published a statement strongly recommending reducing overall anticholinergic burden and consideration of alternatives for OAB [27]. With similar concerns for the NGB population, this pilot trial provides evidence for the feasibility of home bladder neuromodulation for NGB in SCI with TTNS, a nonpharmacologic alternative treatment without anticholinergic side effects.

Subjects in the trial found TTNS easy to use and were nearly $100 \%$ compliant for 4 weeks of daily use. It is unlikely that daily use will be needed in sustained management of TTNS, as weekly use may only be needed for maintenance [26]. Satisfaction with TTNS was very high, with nearly everyone strongly recommending TTNS for those with NGB, and would prefer using TTNS if equal to their bladder medication efficacy. Also, participants responded that the use of TTNS was not embarrassing, not surprising considering the ankle is a modest site for electric stimulation should assistance be required from caregivers. All
16 participants indicated that they wanted to continue using TTNS at home after conclusion of their study participation.

The majority of subjects were able to maintain reduced anticholinergic bladder medications by the end of the 4-week trial of TTNS. This explains the decreased anticholinergic side effects at the end of the trial, with significant reductions in dry mouth and feeling tired. In the treatment of $\mathrm{OAB}$, reducing anticholinergic side effects have been recognized as a way to improve QoL [28]. This may have contributed to the increased IQoL scores observed in this trial.

This trial also suggests preliminary evidence of efficacy, without significant worsening of catheterization volumes and frequency, while reducing anticholinergic bladder medications. Although not significant, frequency of reported incontinence increased during the trial. One possible reason for this increased incontinence is that it may reflect the increased incontinence in those with UTIs. Another possible explanation is that patients with 2 bladder medications experienced worse inconti- 
nence while reducing their medications. In practice, these patients likely tried several medications and combinations to manage incontinence as their bladder compliance and capacity deteriorated. It is unlikely that the mechanism of TTNS can help with improving compliance and capacity after the bladder has significantly remodeled.

The leading theory for the mechanism of bladder neuromodulation in TTNS is that stimulation of the tibial nerve sensory afferents blocks the visceral bladder afferents at the level of the spinal cord [29]. This leads to a disruption of the bladder reflexive arc, decreasing the motor efferent activity and reflexive bladder contractions, reducing episodes of DO and DSD. It may be that the mechanism of TTNS is insufficient to prevent DO and DSD in cases of severely deteriorated NGB, highlighting the need to prevent this development. TTNS has been explored in acute SCI with the hypothesis that early intervention may mitigate the morbidity that develops in the natural course of SCI NGB [24].

There are several limitations in this study, primarily the lack of a sham-control group and urodynamic outcome measures, limiting conclusions on efficacy outcomes. However, this pilot trial served the primary purpose of determining the feasibility of TTNS as a home treatment plan for SCI NGB and provides information on treatment effects. Future studies incorporating urodynamic studies would be required to establish TTNS as an acceptable method for NGB management in SCI. Also, the small number of participants, the duration of the intervention, and the lack of long-term follow-up are limitations, however this was a pilot trial with the goal of generating data for a larger study. Finally, participants in this cohort were using typical first-line bladder anticholinergic medications, not beta-3 adrenergic receptor agonists, which have been shown to reduce NGB symptom burden without improving urodynamic parameters, pad weights, or voiding diary parameters compared to placebo [30]. However, this class of medication is not currently FDA-approved for use in NGB.

In conclusion, this pilot trial suggests self-administration of TTNS in chronic SCI is feasible to perform at home for the management of NGB. In a population known to require higher doses of bladder medications resulting in high rates of noncompliance, an accessible, cost-effective, nonpharmacologic treatment option is needed. Studies with long-term outcomes and urodynamic measures are needed to establish TTNS as a standard management option for SCI NGB.

\section{ACKNOWLEDGMENTS}

We thank Vanessa Bernal, CCRP, Research Coordinator II, for her assistance with TTNS and diligence with contacting research subjects and collecting data.

\section{AUTHOR CONTRIBUTION STATEMENT}

- Full access to all the data in the study and takes responsibility for the integrity of the data and the accuracy of the data analysis: $A S$

- Study concept and design: $A S$

- Acquisition of data: $A S$

- Analysis and interpretation of data: $A S, R K, J E F, S L G$

- Drafting of the manuscript: $A S, R K, J E F, S L G$

- Critical revision of the manuscript for important intellectual content: AS, RK, JEF, SLG

- Statistical analysis: $A S$

- Obtained funding: $A S$

- Administrative, technical, or material support: $A S$

-Study supervision: $A S$

\section{REFERENCES}

1. Ackery A, Tator C, Krassioukov A. A global perspective on spinal cord injury epidemiology. J Neurotrauma 2004;21:1355-70.

2. Weld KJ, Dmochowski RR. Association of level of injury and bladder behavior in patients with post-traumatic spinal cord injury. Urology 2000;55:490-4.

3. Anderson KD. Targeting recovery: priorities of the spinal cord-injured population. J Neurotrauma 2004;21:1371-83.

4. Anderson KD. Session 3: with us, not for us: community activity and priorities. SCI 2020: launching a decade for disruption in spinal cord injury research. Bethesda (MD): NIH Natcher Conference Center; 2019.

5. Bacsu CD, Chan L, Tse V. Diagnosing detrusor sphincter dyssynergia in the neurological patient. BJU Int 2012;109 Suppl 3:31-4.

6. Consortium for Spinal Cord Medicine. Bladder management for adults with spinal cord injury: a clinical practice guideline for health-care providers. J Spinal Cord Med 2006;29:527-73.

7. del Popolo G, Mencarini M, Nelli F, Lazzeri M. Controversy over the pharmacological treatments of storage symptoms in spinal cord injury patients: a literature overview. Spinal Cord 2012;50:8-13.

8. Stöhrer M, Blok B, Castro-Diaz D, Chartier-Kastler E, Del Popolo G, Kramer G, et al. EAU guidelines on neurogenic lower urinary 
tract dysfunction. Eur Urol 2009;56:81-8.

9. Gray SL, Anderson ML, Dublin S, Hanlon JT, Hubbard R, Walker $\mathrm{R}$, et al. Cumulative use of strong anticholinergics and incident dementia: a prospective cohort study. JAMA Intern Med 2015;175: 401-7.

10. Risacher SL, McDonald BC, Tallman EF, West JD, Farlow MR, Unverzagt FW, et al. Association between anticholinergic medication use and cognition, brain metabolism, and brain atrophy in cognitively normal older adults. JAMA Neurol 2016;73:721-32.

11. Fougere RJ, Currie KD, Nigro MK, Stothers L, Rapoport D, Krassioukov AV. Reduction in bladder-related autonomic dysreflexia after onabotulinumtoxinA treatment in spinal cord injury. J Neurotrauma 2016;33:1651-7.

12. Cruz F, Herschorn S, Aliotta P, Brin M, Thompson C, Lam W, et al. Efficacy and safety of onabotulinumtoxinA in patients with urinary incontinence due to neurogenic detrusor overactivity: a randomised, double-blind, placebo-controlled trial. Eur Urol 2011;60: 742-50.

13. BOTOX (onabotulinumtoxinA) [package insert]. Irvine (CA): Allergan Pharmaceuticals Ireland; 2011.

14. Tanagho EA, Schmidt RA. Bladder pacemaker: scientific basis and clinical future. Urology 1982;20:614-9.

15. Tanagho EA, Schmidt RA, Orvis BR. Neural stimulation for control of voiding dysfunction: a preliminary report in 22 patients with serious neuropathic voiding disorders. J Urol 1989;142(2 Pt 1):3405.

16. Sievert KD, Amend B, Gakis G, Toomey P, Badke A, Kaps HP, et al. Early sacral neuromodulation prevents urinary incontinence after complete spinal cord injury. Ann Neurol 2010;67:74-84.

17. McGuire EJ, Zhang SC, Horwinski ER, Lytton B. Treatment of motor and sensory detrusor instability by electrical stimulation. J Urol 1983;129:78-9.

18. Canbaz Kabay S, Kabay S, Mestan E, Cetiner M, Ayas S, Sevim M, et al. Lvong term sustained therapeutic effects of percutaneous posterior tibial nerve stimulation treatment of neurogenic overactive bladder in multiple sclerosis patients: 12-months results. Neurourol Urodyn 2017;36:104-10.

19. Gupta P, Ehlert MJ, Sirls LT, Peters KM. Percutaneous tibial nerve stimulation and sacral neuromodulation: an update. Curr Urol Rep 2015;16:4

20. Gaziev G, Topazio L, Iacovelli V, Asimakopoulos A, Di Santo A, De
Nunzio C, et al. Percutaneous Tibial Nerve Stimulation (PTNS) efficacy in the treatment of lower urinary tract dysfunctions: a systematic review. BMC Urol 2013;13:61.

21. Sirls ER, Killinger KA, Boura JA, Peters KM. Percutaneous tibial nerve stimulation in the office setting: real-world experience of over 100 patients. Urology 2018;113:34-9.

22. Chen G, Liao L, Li Y. The possible role of percutaneous tibial nerve stimulation using adhesive skin surface electrodes in patients with neurogenic detrusor overactivity secondary to spinal cord injury. Int Urol Nephrol 2015;47:451-5.

23. de Sèze M, Raibaut P, Gallien P, Even-Schneider A, Denys P, Bonniaud $\mathrm{V}$, et al. Transcutaneous posterior tibial nerve stimulation for treatment of the overactive bladder syndrome in multiple sclerosis: results of a multicenter prospective study. Neurourol Urodyn 2011; 30:306-11.

24. Stampas A, Korupolu R, Zhu L, Smith CP, Gustafson K. Safety, feasibility, and efficacy of transcutaneous tibial nerve stimulation in acute spinal cord injury neurogenic bladder: a randomized control pilot trial. Neuromodulation 2019;22:716-22.

25. Amarenco G, Ismael SS, Even-Schneider A, Raibaut P, DemailleWlodyka S, Parratte B, et al. Urodynamic effect of acute transcutaneous posterior tibial nerve stimulation in overactive bladder. J Urol 2003;169:2210-5.

26. Finazzi Agrò E, Campagna A, Sciobica F, Petta F, Germani S, Zuccalà $\mathrm{A}$, et al. Posterior tibial nerve stimulation: is the once-a-week protocol the best option? Minerva Urol Nefrol 2005;57:119-23.

27. American Urogynecologic Society (AUGS) Guidelines Committee. AUGS consensus statement: association of anticholinergic medication use and cognition in women with overactive bladder. Female Pelvic Med Reconstr Surg 2017;23:177-8.

28. Kim A, Lee KS, Jung R, Na S, Kim JC, Kim HG, et al. Health related quality of life in patients with side-effects after antimuscarinic treatment for overactive bladder. Low Urin Tract Symptoms 2017; 9:171-5.

29. Sanford MT, Suskind AM. Neuromodulation in neurogenic bladder. Transl Androl Urol 2016;5:117-26.

30. Welk B, Hickling D, McKibbon M, Radomski S, Ethans K. A pilot randomized-controlled trial of the urodynamic efficacy of mirabegron for patients with neurogenic lower urinary tract dysfunction. Neurourol Urodyn 2018;37:2810-7. 